\title{
Thermal Effect on Free Vibration of Non-Homogeneous Orthotropic Visco-Elastic Rectangular Plate of Parabolically Varying Thickness
}

\author{
Arun Kumar Gupta, Pooja Singhal \\ Department of Mathematics, M. S. College, Saharanpur, India \\ E-mail:gupta_arunnitin@yahoo.co.in,poojaacad@yahoo.in \\ Received August 31, 2010; revised September 17, 2010; accepted September 21, 2010
}

\begin{abstract}
A simple model presented here is to study the thermal effect on vibration of non-homogeneous orthotropic visco-elastic rectangular plate of parabolically varying thickness having clamped boundary conditions on all the four edges. For non-homogeneity of the plate material, density is assumed to vary linearly in one direction. Using the separation of variables method, the governing differential equation has been solved for vibration of non-homogeneous orthotropic visco-elastic rectangular plate. An approximate frequency equation is derived by using Rayleigh-Ritz technique with a two-term deflection function. Results are calculated for time period and deflection at different points, for the first two modes of vibration, for various values of temperature gradients, non-homogeneity constant, taper constant and aspect ratio and shown by graphs.
\end{abstract}

Keywords: Thermal Gradient, Vibration, Non-Homogeneous, Orthotropic, Visco-Elastic, Rectangular Plate, Variable Thickness

\section{Introduction}

In modern technology an interest towards the effect of high temperatures on non-homogeneous plates of variable thickness is developed due to applications in various engineering branches such as nuclear, power plants, aero-nautical, chemical etc. where metals and their alloys exhibits visco-elastic behavior. Therefore for these changes the structures are exposed to high intensity, heat fluxes and material properties undergo significant changes.

The materials are being developed, depending upon the requirement and durability, so that these can be used to give better strength, flexibility, weight effectiveness and efficiency. So some new materials and alloys are utilized in making structural parts of equipment used in modern technological industries like space craft, jet engine, earth quake resistance structures, telephone industry etc. Applications of such materials are due to reduction of weight and size, low expenses and enhancement in effectiveness and strength. It is well known that first few frequencies of structure should be known before finalizing the design of a structure. The study of vibration of plate structures is important in a wide variety of applications in engineering design. Elastic plates are widely employed nowadays in civil, aeronautical and marine structures designs. Complex shapes with variety of thickness variation are sometimes incorporated to reduce costly material, lighten the loads, and provide ventilation and to alter the resonant frequencies of the structures. Dynamic behavior of these structures is strongly dependent on boundary conditions, geometric shapes, material properties etc.

As technology develops new discoveries day by day like in jet engine, field of spacecraft and nuclear power plants etc., the time dependent behavior of materials has become of great importance. Thus, the need of the study of vibration of visco-elastic plates (it may be rectangular, circular, elliptical etc.) of certain aspect ratios with some simple boundary conditions has been increased rapidly.

Vibration phenomenon, common in mechanical devices and structures, is undesirable in many cases, such as machine tools. But this phenomenon is not always unwanted; for example, vibration is needed in the operation of vibration screens.

In the course of time, engineers have become increasingly conscious of the importance of an elastic behavior of many materials and mathematical formulations have 
been attempted and applied to practical problems. Since plastics and new materials are widely used in the construction of equipment and structures, so the development of the application of visco-elasticity is needed to permit rational design.

Leissa's monograph [1] contains an excellent discussion of the subject of vibrating plates with elastic edge support. Leissa [2] has given the solution for rectangular plate of variable thickness. Gupta, Johri and Vats [3] have discussed the thermal effect on vibration of non- homogeneous orthotropic rectangular plate having bi- directional parabolically varying thickness. Sobotka [4] discussed the free vibration of visco-elastic orthotropic rectangular plates were discussed. Tomar and Gupta [5-7] solved the vibration problem of orthotropic rectangular plate of varying thickness subjected to a thermal gradient. Lal [8] has studied the transverse vibrations of orthotropic non-uniform rectangular plates with continuously varying density.

Visco-elasticity, as its name implies, is a generalization of elasticity and viscosity. The ideal linear elastic element is the spring. When a tensile force is applied to it, the increase in distance between its two ends is proportional to the force. The ideal linear viscous element is the dashpot.

The plate type structural components in aircraft and rockets have to operate under elevated temperatures that cause non-homogeneity in the plate material, i.e., elastic constants of the materials becomes functions of space variables. In an up-to-date survey of literature, authors have come across various models to account for nonhomogeneity of plate materials proposed by researchers dealing with vibration but none of them consider non-homogeneity with thermal effect on visco-elastic plates.

Gupta and Khanna [9] have studied the effect of linearly varying thickness in both directions on vibration of visco-elastic rectangular plate. Gupta and Kaur [10] studied the effect of thermal on vibration of clamped viscoelastic rectangular plate with linearly thickness in both directions. Gupta, Kumar and Gupta [11] have considered the vibration of visco-elastic parallelogram plate with parabolic thickness variation. Recently, Gupta and Singhal [12] discussed the effect of non-homogeneity on thermally induced vibration of orthotropic visco-elastic rectangular plate of linearly varying thickness.

The object of the present study is to determine the thermal effect on vibration of non-homogeneous orthotropic visco-elastic rectangular plate of parabolically varying thickness. It is clamped supported on all the four edges. The assumption of small deflection and linear orthotropic visco-elastic properties are made. It is further assumed that the visco-elastic properties of the plates are of the Kelvin type. Time period and deflection for the first two modes of vibration are calculated for the various values of thermal gradients, non-homogeneity constant, aspect ratio and taper constant.

\section{Analysis}

The equation of motion of a visco-elastic rectangular plate of variable thickness is [12]:

$\left[\begin{array}{l}D_{x} \frac{\partial^{4} W}{\partial x^{4}}+D_{y} \frac{\partial^{4} W}{\partial y^{4}}+2 H \frac{\partial^{4} W}{\partial x^{2} \partial y^{2}}+ \\ 2 \frac{\partial H}{\partial x} \frac{\partial^{3} W}{\partial x \partial y^{2}}+2 \frac{\partial H}{\partial y} \frac{\partial^{3} W}{\partial x^{2} \partial y}+2 \frac{\partial D_{x}}{\partial x} \frac{\partial^{3} W}{\partial x^{3}}+ \\ 2 \frac{\partial D_{y}}{\partial y} \frac{\partial^{3} W}{\partial y^{3}}+\frac{\partial^{2} D_{x}}{\partial x^{2}} \frac{\partial^{2} W}{\partial x^{2}}+\frac{\partial^{2} D_{y}}{\partial y^{2}} \frac{\partial^{2} W}{\partial y^{2}}+ \\ \frac{\partial^{2} D_{1}^{\prime}}{\partial x^{2}} \frac{\partial^{2} W}{\partial y^{2}}+\frac{\partial^{2} D_{1}^{\prime}}{\partial y^{2}} \frac{\partial^{2} W}{\partial x^{2}}+4 \frac{\partial^{2} D_{x y}}{\partial x \partial y} \frac{\partial^{2} W}{\partial x \partial y}\end{array}\right]-\rho h p^{2} W=0$

and

$$
\ddot{T}+p^{2} \tilde{D} T=0
$$

where Equations (1) and (2) are the differential equations of motion for orthotropic plate of variable thickness and time function for visco-elastic orthotropic plate for free vibration respectively.

Here $p^{2}$ is a constant and

$$
H=D_{1}^{\prime}+2 D_{x y}, \quad D_{x}=\frac{E_{x} h^{3}}{12\left(1-v_{x} v_{y}\right)},
$$

is called the flexural rigidity of the plate in $x$-direction,

$$
D_{y}=\frac{E_{y} h^{3}}{12\left(1-v_{x} v_{y}\right)},
$$

is called the flexural rigidity of the plate in $y$-direction,

$$
D_{x y}=\frac{G_{x y} h^{3}}{12},
$$

is called the torsion rigidity,

$D_{1}^{\prime}=v_{x} D_{y}\left(=v_{y} D_{x}\right), \tilde{D}$ is Rheological operator and $E_{x} \& E_{y}$ are the modules of elasticity in $\mathrm{x}$ - and y-direction respectively, $v_{x}$ and $v_{y}$ are the Poisson ratios \& $G_{x y}$ is the shear modulus.

Assuming steady one dimensional temperature distribution along the length, i.e., $x$-direction for the plate as

$$
\tau=\tau_{0}\left(1-\frac{\mathrm{x}}{\mathrm{a}}\right)
$$


where $\tau$ denotes the temperature excess above the reference temperature at any point at distance $x / a$ and $\tau_{0}$ denotes the temperature excess above reference temperature at the end, i.e., $\mathrm{x}=\mathrm{a}$.

Temperature dependence of the modulus of elasticity for most of engineering materials can be expressed in this form

$$
E_{x}=E_{1}(1-\gamma \tau), E_{y}=E_{2}(1-\gamma \tau), G_{x y}=G_{0}(1-\gamma \tau)
$$

Here $E_{1}$ and $E_{2}$ are values of the Young's moduli respectively along the $x$ and $y$ axis at the reference temperature, i.e., at $\tau=0$ and $\gamma$ is the slope of the variation of modulus of elasticity with $\tau$.

Thus modulus variation become

$$
\begin{gathered}
E_{x}(x)=E_{1}[1-\alpha(1-x / a)], \\
E_{y}(x)=E_{2}[1-\alpha(1-x / a)], \\
G_{x y}(x)=G_{0}[1-\alpha(1-x / a)]
\end{gathered}
$$

where $\alpha=\gamma \tau_{0}(0 \leq \alpha \leq 1)$, a parameter ,known as thermal gradient.

The expression for the strain energy $V$ and Kinetic energy $P$ in the plate are [1].

$$
\begin{gathered}
V=\frac{1}{2} \int_{0}^{a} \int_{0}^{b}\left[D_{x}\left(W_{, x x}\right)^{2}+D_{y}\left(W_{, y y}\right)^{2}+\right. \\
\left.2 D_{1} W_{, x x} W_{, y y}+4 D_{x y}\left(W_{, x y}\right)^{2}\right] d x d y \\
P=\frac{1}{2} p^{2} \int_{0}^{a} \int_{0}^{b} \rho h W^{2} d x d y
\end{gathered}
$$

Assuming thickness and density varies parabolically and linearly in the $x$-direction respectively, therefore one can take

$$
\begin{aligned}
h & =h_{0}\left\{1+\beta(x / a)^{2}\right\} \\
\text { and } \quad & \rho=\rho_{0}\left(1+\alpha_{1} x / a\right)
\end{aligned}
$$

where $\beta$ is the taper constant and $\alpha_{1}$ is non-homogeneity constant.

\section{Solution and Frequency Equation}

To find a solution, we use Rayleigh-Ritz technique. This method requires that maximum strain energy must be equal to the maximum kinetic energy. So, it is necessary for the problem under consideration that

$$
\delta(V-P)=0
$$

for arbitrary variations of $W$ satisfying relevant geometrical boundary conditions which are

$$
\begin{array}{lll}
W=W_{x}=0 & \text { at } & x=0, a \\
W=W_{y}=0 & \text { at } & y=0, b
\end{array}
$$

and the corresponding two term deflection function is taken as [12].

$$
\begin{aligned}
& W=[(x / a)(y / b)(1-x / a)(1-y / b)]^{2} \\
& {\left[A_{1}+A_{2}(x / a)(y / b)(1-x / a)(1-y / b)\right]}
\end{aligned}
$$

The non-dimensional variables are

$$
\begin{gathered}
X=x / a, Y=y / b, \bar{W}=W / a, \bar{h}=h / a, \bar{\rho}=\rho / a \\
E_{1}{ }^{*}=E_{1} /\left(1-v_{x} v_{y}\right), E_{2}{ }^{*}=E_{2} /\left(1-v_{x} v_{y}\right) \\
E^{*}=v_{x} E_{2}{ }^{*}=v_{y} E_{1}^{*}
\end{gathered}
$$

By using Equations (5), (8), (9) and (13) in (6) and (7), one gets

$$
P=\frac{1}{2} \rho_{o} p^{2} \overline{h_{o}} a^{5} \int_{0}^{1} \int_{0}^{b / a}\left[\left(1+\alpha_{1} X\right)\left(1+\beta X^{2}\right) \bar{W}^{2}\right] d X d Y
$$

and

$$
\begin{aligned}
V=R & \int_{0}^{1} \int_{0}^{b / a}\left[\{ 1 - \alpha ( 1 - X ) \} ( 1 + \beta X ^ { 2 } ) ^ { 3 } \left\{\left(\bar{W}_{, X X}\right)^{2}+\right.\right. \\
& \left(E_{2}^{*} / E_{1}^{*}\right)\left(\bar{W}_{, Y Y}\right)^{2}+\left(2 E^{*} / E_{1}^{*}\right) \bar{W}_{, X X} \bar{W}_{, Y Y}+ \\
& \left.\left.\left(4 G_{o} / E_{1}^{*}\right)\left(\bar{W}_{, X Y}\right)^{2}\right\}\right] d X d Y
\end{aligned}
$$

where $R=\frac{1}{2}\left(E_{1}^{*} \bar{h}^{3} / 12\right) a$

On substituting the values of $P$ and $V$ from Equations (14) and (15) in Equation (10), we get

$$
\begin{gathered}
\left(V_{1}-\lambda^{2} p^{2} P_{1}\right)=0 \\
V_{1}=\int_{0}^{1} \int_{0}^{b / a}\left[\{ 1 - \alpha ( 1 - X ) \} ( 1 + \beta X ^ { 2 } ) ^ { 3 } \left\{\left(\bar{W}_{, X X}\right)^{2}+\right.\right. \\
\left(E_{2}^{*} / E_{1}^{*}\right)\left(\bar{W}_{, Y Y}\right)^{2}+\left(2 E^{*} / E_{1}^{*}\right) \bar{W}_{, X X} \bar{W}_{, Y Y}+ \\
\left.\left.\left(4 G_{o} / E_{1}^{*}\right)\left(\bar{W}_{, X Y}\right)^{2}\right\}\right] d X d Y \\
P_{1}=\int_{0}^{1} \int_{0}^{b / a}\left[\left(1+\alpha_{1} X\right)\left(1+\beta X^{2}\right) \bar{W}^{2}\right] d X d Y
\end{gathered}
$$

where $\lambda^{2}=\frac{12 a^{4} \rho_{o}}{E_{1}^{*}{\overline{h_{o}}}^{2}}$

Equation (17) involves the unknown $A_{1}$ and $A_{2}$ arising due to the substitution of $W(x, y)$ from Equation (12). These two constants are to be determined from Equation (17) as follows: 
$\frac{\partial}{\partial \mathrm{A}_{\mathrm{n}}}\left(\mathrm{V}_{1}-\lambda^{2} \mathrm{p}^{2} \mathrm{P}_{1}\right)=0$ where $n=1,2$

On simplifying (21) we get

$$
b_{n 1} A_{1}+b_{n 2} A_{2}=0
$$

where $n=1,2, b_{n 1}, b_{n 2}$ involves parametric constants and the frequency parameter $p$. For a non-trivial solution, the determinant of the coefficient of Equation (22) must be zero. So, we get the frequency equation as

$$
\left|\begin{array}{ll}
b_{11} & b_{12} \\
b_{21} & b_{22}
\end{array}\right|=0
$$

On solving Equation (23) one gets a quadratic equation in $p^{2}$, which gives two values of $p^{2}$. On substituting the value of $A_{1}=1$, by choice, in Equation (12) one get $A_{2}=-b_{11} / b_{12}$ and hence $W$ becomes:

$$
\begin{gathered}
W=\left[X Y \frac{a}{b}(1-X)\left(1-Y \frac{a}{b}\right)\right]^{2} \\
{\left[1+\left(-\frac{b_{11}}{b_{12}}\right) X Y\left(\frac{a}{b}\right)(1-X)\left(1-Y \frac{a}{b}\right)\right]}
\end{gathered}
$$

\section{Time Functions of Vibration of Viscoelastic Plates}

Equation (2) is defined as general differential equation of time functions of free vibrations of visco-elastic orthotropic plates. It depends on visco-elastic operator $\tilde{D}$. One has, for Kelvin's model [12],

$$
\tilde{D} \equiv\left\{1+\left(\frac{\eta}{G}\right)\left(\frac{d}{d t}\right)\right\}
$$

where $\eta$ is visco-elastic constant and $G$ is shear modulus. Taking temperature dependence of viscoelastic constant $\eta$ and shear modulus $G$ is the same form as that of Young's moduli, we have

$$
G(\tau)=G_{0}\left(1-\gamma_{1} \tau\right), \quad \eta(\tau)=\eta_{0}\left(1-\gamma_{2} \tau\right)
$$

where $G_{0}$ is shear modulus and $\eta_{0}$ is visco-elastic constant at some reference temperature, i.e., at $\tau=0, \gamma_{1}$ and $\gamma_{2}$ are slope variation of $\tau$ with $G$ and $\eta$ respectively. Substituting the value of $\tau$ from Equation (3) and using Equation (13) in Equation (26), one gets:

$$
\begin{aligned}
& G=G_{0}\left[1-\alpha_{5}(1-X)\right], \text { where } \alpha_{5}=\gamma_{1} \tau_{0}, \\
& 0 \leq \alpha_{5} \leq 1 \text { and } \eta=\eta_{0}\left[1-\alpha_{4}(1-X)\right], \\
& \text { where } \alpha_{4}=\gamma_{2} \tau_{0}, \quad 0 \leq \alpha_{4}<1
\end{aligned}
$$

Here $\alpha_{4}$ and $\alpha_{5}$ are thermal constants.

After using Equation (25) in Equation (2), one obtains

$$
\ddot{T}+p^{2} k \dot{T}+p^{2} T=0
$$

where $k=\frac{\eta}{G}=\frac{\eta_{0}\left[1-\alpha_{4}(1-X)\right]}{G_{0}\left[1-\alpha_{5}(1-X)\right]}$

Equation (28) is a differential equation of second order for time function $T$.

Solution of Equation (28) will be

$$
T(t)=e^{a_{1} t}\left[C_{1} \cos b_{1} t+C_{2} \sin b_{1} t\right]
$$

where $a_{1}=-p^{2} k / 2$

$$
b_{1}=p \sqrt{1-\left(\frac{p k}{2}\right)^{2}}
$$

and $C_{1}, C_{2}$ are constants which can be determined easily from initial conditions of the plate.

Assuming initial conditions as

$$
T=1 \text { and } \dot{T}=0 \text { at } t=0
$$

Using Equation (33) in Equation (30), one obtains

$$
C_{1}=1 \& C_{2}=-a_{1} / b_{1}
$$

One has

$$
T(t)=e^{a_{1} t}\left[\cos b_{1} t+\left(-a_{1} / b_{1}\right) \sin b_{1} t\right]
$$

after using Equation (34) in Equation (30).

Thus, deflection of vibrating mode $w(x, y, t)$, which is equal to $W(x, y) T(t)$, may be expressed as

$$
\begin{gathered}
w=[X Y(a / b)(1-X)(1-Y a / b)]^{2} \\
{\left[1+\left(-b_{11} / b_{12}\right) X Y(a / b)(1-X)(1-Y a / b)\right] \times} \\
{\left[e^{a_{1} t}\left\{\cos b_{1} t+\left(-a_{1} / b_{1}\right) \sin b_{1} t\right\}\right]}
\end{gathered}
$$

by using Equations (24) \& Equation (35).

Time period of the vibration of the plate is given by

$$
K=2 \pi / p
$$

where $p$ is the frequency given by Equation (23).

\section{Numerical Evaluations}

The values of time period $(K)$ and deflection $(w)$ (at two different instant of time) for a clamped visco-elastic orthotropic non-homogeneous rectangular plate for different values of taper constant $\beta$, thermal constants $\left(\alpha, \alpha_{4}\right.$, $\alpha_{5}$ ), non homogeneity constant $\alpha_{1}$ and aspect ratio $a / b$ at different points for first two modes of vibrations are calculated.

The following orthotropic material parameters have been taken as [1].

$$
E_{2}^{*} / E_{1}^{*}=0.32
$$




$$
\begin{gathered}
E^{*} / E_{1}^{*}=0.04 \\
G_{o} / E_{1}^{*}=0.09 \\
\eta_{o} / G_{o}=0.000069 \\
\rho_{o}=3 \times 10^{5}
\end{gathered}
$$

(mass density per unit volume of the plate material).

The thickness of the plate at the centre is taken as $h_{o}=$ 0.01 meter.

\section{Results and Discussion}

Numerical results for a visco-elastic orthotropic non-homogeneous rectangular plate of parabolically varying thickness have been computed with accuracy by using latest computer technology. Computations have been made for calculating time period $K$ and deflection $w$ (at two different instant of time) for different values of taper constant $\beta$, thermal constants $\left(\alpha, \alpha_{4}, \alpha_{5}\right)$, non homogeneity constant $\alpha_{1}$ and aspect ratio $a / b$ for first two modes of vibration. All these results are presented in Figure 1 to Figure 7. Comparison is made with the author's paper [12] for uniform plate and found to be in very close agreement.

Figure 1 shows the result of time period $K$ for first two modes of vibration for different values of taper constant $\beta$ and fixed aspect ratio $a / b=1.5$ and four combinations of non-homogeneity constant $\alpha_{1}$ and thermal constant $\alpha$ are

$$
\begin{aligned}
& \alpha_{1}=0.0, \alpha=0.0 \\
& \alpha_{1}=0.0, \alpha=0.2 \\
& \alpha_{1}=0.4, \alpha=0.0
\end{aligned}
$$

$$
\alpha_{1}=0.4, \alpha=0.2
$$

It can be seen that time period $\mathrm{K}$ decreases when taper constant increases for first two modes of vibration.

Figure 2 shows the result of time period $K$ for first two modes of vibration for different values of non-homogeneity constant $\alpha_{1}$ and fixed aspect ratio $a / b=$ 1.5 and four combinations of taper constant $\beta$ and thermal constant $\alpha$ are

$$
\begin{aligned}
& \beta=0.0, \alpha=0.0 \\
& \beta=0.4, \alpha=0.0 \\
& \beta=0.0, \alpha=0.2 \\
& \beta=0.4, \alpha=0.2
\end{aligned}
$$

It can be seen that time period $K$ increases when nonhomogeneity constant increases for first two modes of vibration.

Figure 3 shows the result of time period $K$ for different aspect ratio and four combinations of thermal constant $\alpha$, taper constant $\beta$ and non-homogeneity constant $\alpha_{1}$, i.e.,

$$
\begin{aligned}
& \alpha=0.2, \beta=0.0, \alpha_{1}=0.0 \\
& \alpha=0.2, \beta=0.4, \alpha_{1}=0.0 \\
& \alpha=0.2, \beta=0.0, \alpha_{1}=0.4 \\
& \alpha=0.2, \beta=0.4, \alpha_{1}=0.4
\end{aligned}
$$

It can be seen that time period $K$ decreases when aspect ratio increases for first two modes of vibration.

Figures 4-7 shows the results of deflection for first two modes of vibration for different $X, Y$ and fixed aspect ratio $a / b=1.5$ for initially time $0 . K$ and time $5 . K$ for the following combination of thermal constants $\left(\alpha, \alpha_{4}\right.$, $\alpha_{5}$ ), taper constant $\beta$ and non-homogeneity constant $\alpha_{1}$.
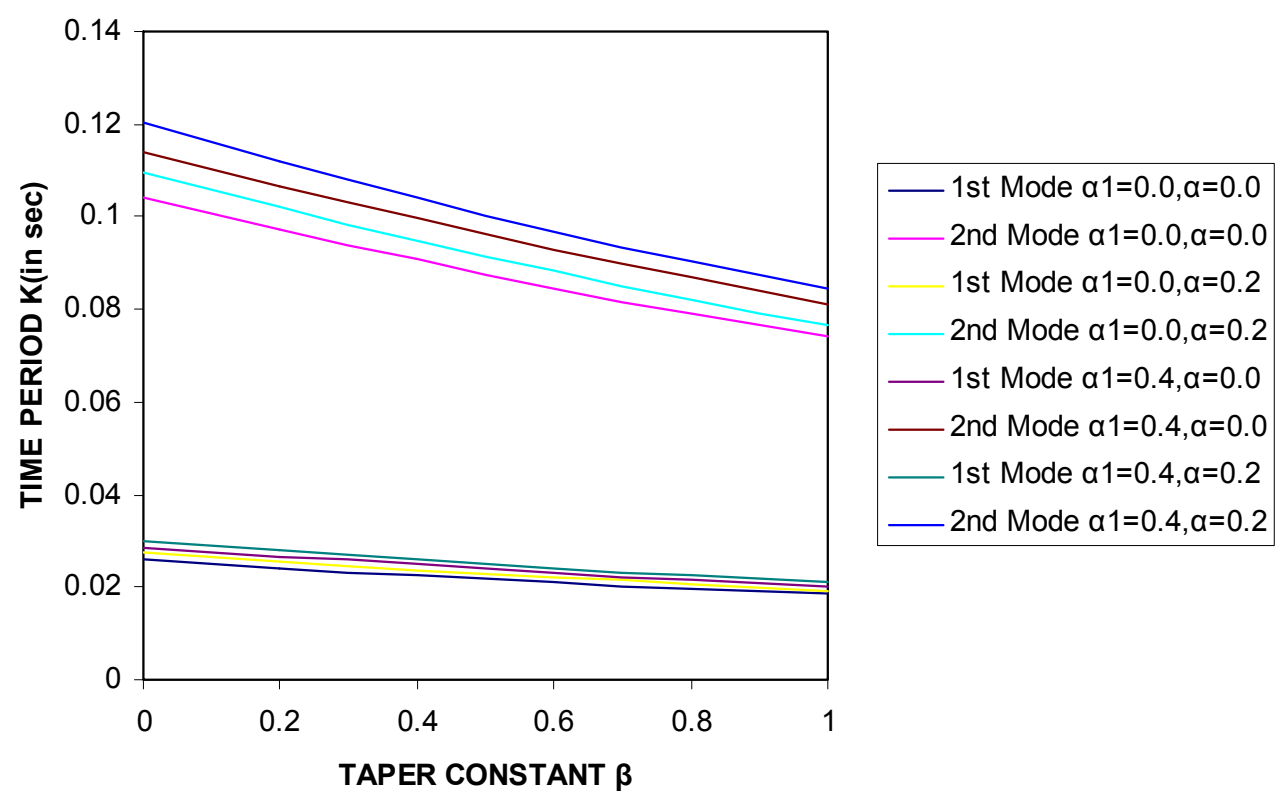

Figure 1. Variation of time period with taper constant of visco-elastic non-homogeneous rectangular plate of parabolically varying thickness. 

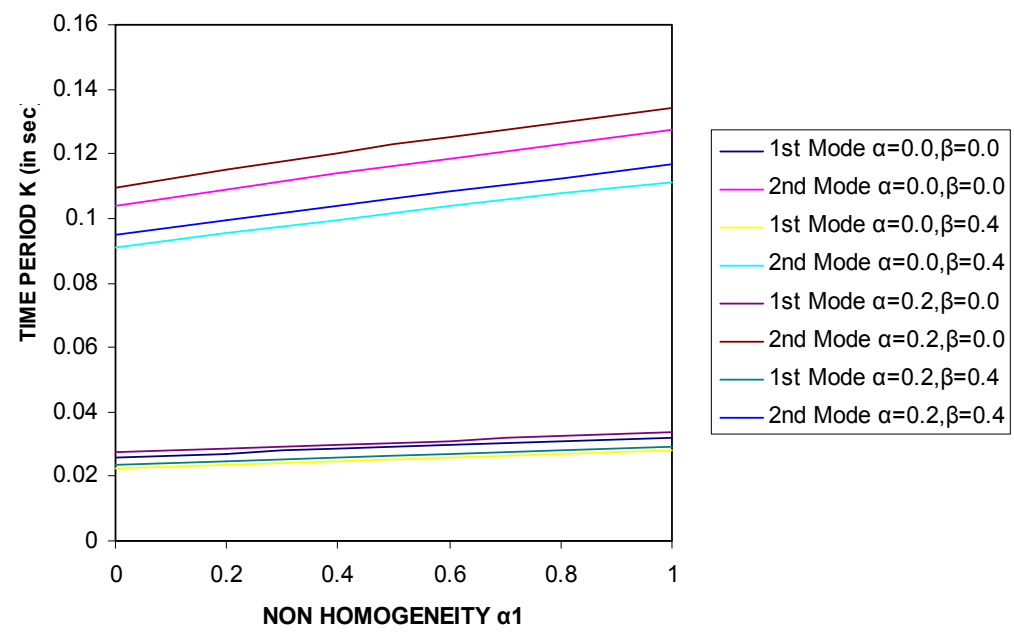

Figure 2. Variation of time period with non-homogeneity constant of visco-elastic non-homogeneous rectangular plate of parabolically varying thickness.

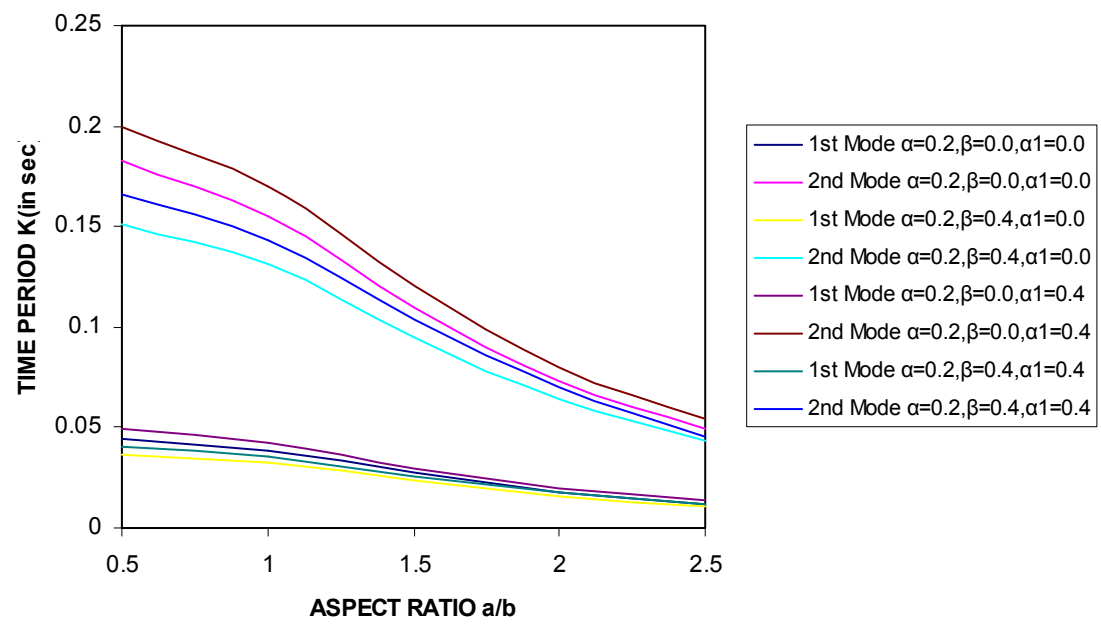

Figure 3. Variation of time period with aspect ratio of visco-elastic non-homogeneous rectangular plate of parabolically varying thickness.

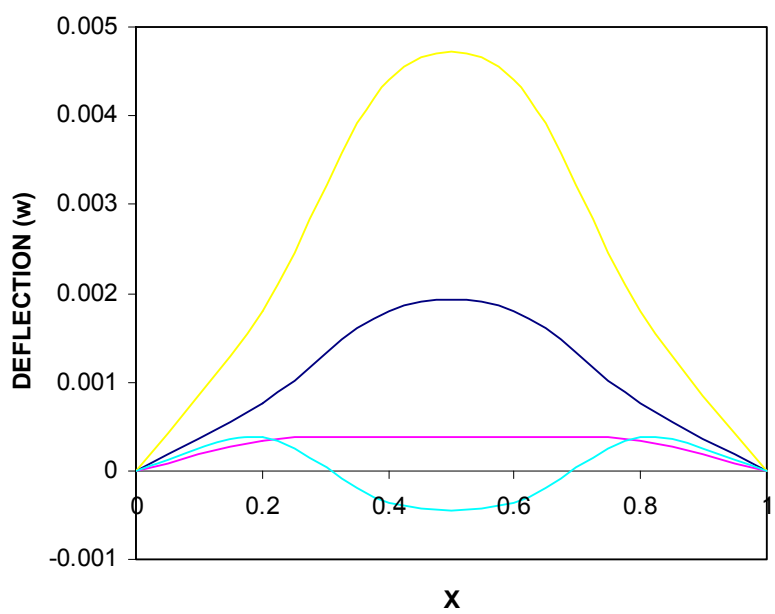

1st Mode $Y=0.2 \mathrm{bla} \mathrm{\&}$
$Y=0.8 \mathrm{bla}$
2nd Mode $Y=0.2 \mathrm{bla} \mathrm{\&}$
$\mathrm{Y}=0.8 \mathrm{bla}$
1st Mode $Y=0.4 \mathrm{bla} \mathrm{\&}$
$\mathrm{Y}=0.6 \mathrm{bla}$
2nd Mode $Y=0.4 \mathrm{bla} \mathrm{\&}$
$Y=0.6 \mathrm{bla}$

Figure 4. Transverse deflection $w V / S X$ of visco-elastic non-homogeneous rectangular plate of parabolically varying thickness at initial time 0 . $K$ having constants combination as $\alpha=0.0, \beta=0.6, \alpha_{1}=0.0, \alpha_{4}=0.3, \alpha_{5}=0.2$. 


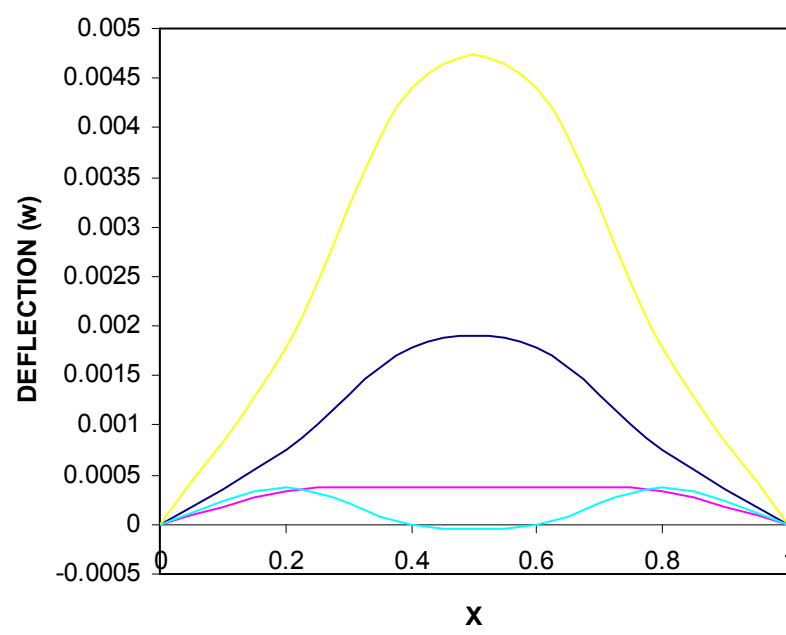

1st Mode $Y=0.2 \mathrm{bla} \mathrm{\&}$
$\mathrm{Y}=0.8 \mathrm{bla}$
2nd Mode $\mathrm{Y}=0.2 \mathrm{bla} \mathrm{\&}$
$\mathrm{Y}=0.8 \mathrm{bla}$
1st Mode $\mathrm{Y}=0.4 \mathrm{bla} \mathrm{\&}$
$\mathrm{Y}=0.6 \mathrm{bla}$
2nd Mode $\mathrm{Y}=0.4 \mathrm{bla} \mathrm{\&}$
$\mathrm{Y}=0.6 \mathrm{bla}$

Figure 5. Transverse deflection $w$ Vs $X$ of visco-elastic non-homogeneous rectangular plate of parabolically varying thickness at initial time $0 . K$ having constants combination as $\alpha=0.0, \beta=0.6, \alpha_{1}=0.0, \alpha_{4}=0.3, \alpha_{5}=0.2$.

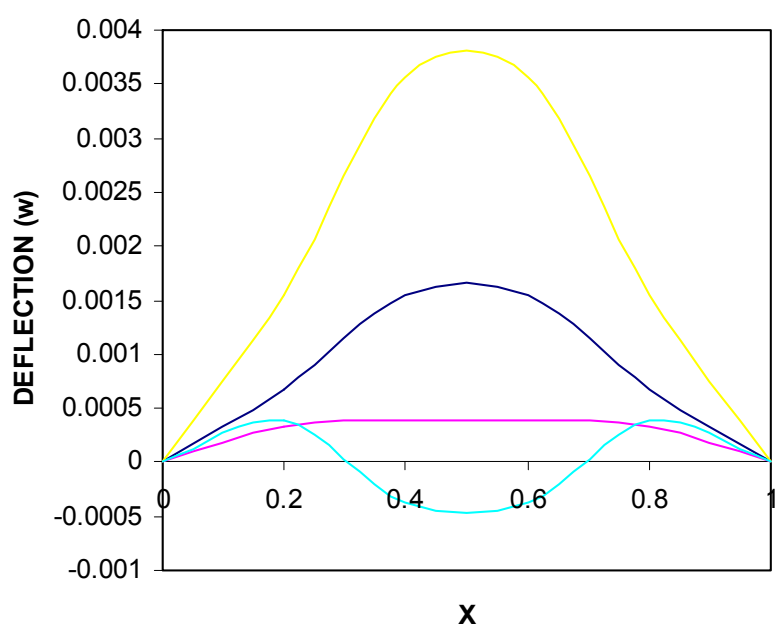

1st Mode $Y=0.2 \mathrm{bla} \mathrm{\&}$
$\mathrm{Y}=0.8 \mathrm{bla}$
2nd Mode $\mathrm{Y}=0.2 \mathrm{bla} \mathrm{\&}$
$\mathrm{Y}=0.8 \mathrm{bla}$
1st Mode $\mathrm{Y}=0.4 \mathrm{bla} \mathrm{\&}$
$\mathrm{Y}=0.6 \mathrm{bla}$
2nd Mode $\mathrm{Y}=0.4 \mathrm{bla} \mathrm{\&}$
$\mathrm{Y}=0.6 \mathrm{bla}$

Figure 6. Transverse deflection $w$ Vs $X$ of visco-elastic non-homogeneous rectangular plate of parabolically varying thickness at time 5. $K$ having constants combination as $\alpha=0.8, \beta=0.0, \alpha_{1}=0.4, \alpha_{4}=0.3, \alpha_{5}=0.2$.

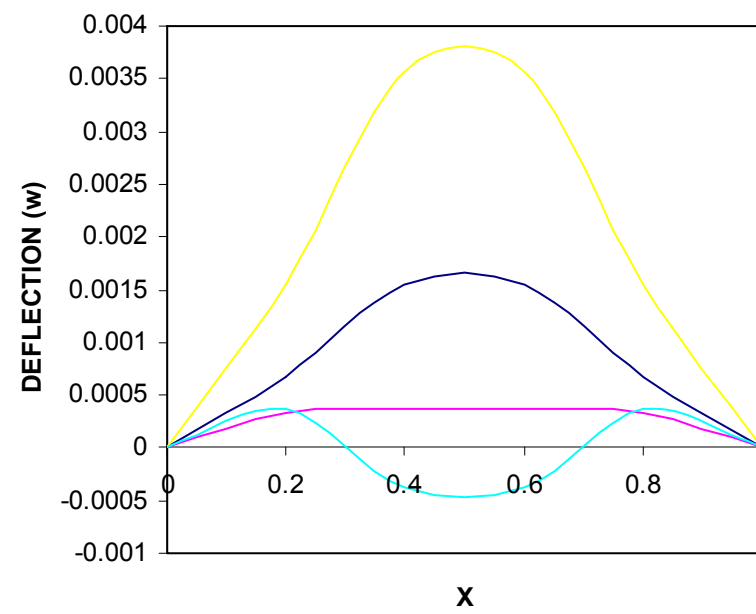

1st Mode $Y=0.2 \mathrm{bla} \mathrm{\&}$
$\mathrm{Y}=0.8 \mathrm{bla}$
2nd Mode $\mathrm{Y}=0.2 \mathrm{bla} \mathrm{\&}$
$\mathrm{Y}=0.8 \mathrm{bla}$
1st Mode $\mathrm{Y}=0.4 \mathrm{bla} \mathrm{\&}$
$\mathrm{Y}=0.6 \mathrm{bla}$
2nd Mode $\mathrm{Y}=0.4 \mathrm{bla} \mathrm{\&}$
$\mathrm{Y}=0.6 \mathrm{bla}$

Figure 7. Transverse deflection $w$ Vs $X$ of visco-elastic non-homogeneous rectangular plate of parabolically varying thickness at time 5. $K$ having constants combination as $\alpha=0.8, \beta=0.0, \alpha_{1}=0.4, \alpha_{4}=0.3, \alpha_{5}=0.2$. 


\section{References}

[1] A. W. Leissa, NASA SP-60, Vibration of Plate, 1969.

[2] A. W. Leissa, "Recent Studies in Plate Vibration 19811985 Part II, Complicating Effects," Shock and Vibration Dig., Vol. 19, No. 3, 1987, pp. 10-24.

[3] A. K. Gupta, T. Johri and R. P. Vats, "Thermal Effect on Vibration of Non-Homogeneous Orthotropic Rectangular Plate Having Bi-Directional Parabolically Varying Thickness," Proceeding of International Conference in World Congress on Engineering and Computer Science, SanFrancisco, October 2007, pp. 784-787.

[4] Z. Sobotka, "Free Vibration of Visco-Elastic Orthotropic Rectangular Plates," Acta Technica, CSAV, Vol. 23, No. 6, 1978, pp. 678-705.

[5] J. S. Tomar and A. K. Gupta, "Effect of Thermal Gradient on Frequencies of Orthotropic Rectangular Plate Whose Thickness Varies in Two Directions," Journal of Sound and Vibration, Vol. 98, No. 2, January 1985, pp. 257-262.

[6] J. S. Tomar and A. K. Gupta, "Thermal Effect on Frequencies of an Orthotropic Rectangular Plate of Linearly Varying Thickness," Journal of Sound and Vibration, Vol. 90, No. 3, October 1983, pp. 325-331.

[7] J. S. Tomar and A. K. Gupta, "Effect of Exponential Temperature Variation on Frequencies of an Orthotropic
Rectangular Plate of Exponentially Varying Thickness,' Proceeding of the Workshop on Computer Application in Continum Mechanics, Roorkee, March 1986, pp. 183188.

[8] R. Lal, "Transverse Vibrations of Orthotropic Non-Uniform Rectangular Plate with Continuously Varying Density," Indian Journal of Pure and Applied Mathematics, Vol. 34, No. 4, 2003, pp. 587-606.

[9] A. K. Gupta and A. Khanna, "Vibration of Visco-Elastic Rectangular Plate with Linearly Thickness Variations in Both Directions," Journal of Sound and Vibration, Vol. 301, No. 3-5, April 2007, pp. 450-457.

[10] A. K. Gupta and H. Kaur, "Study of the Effect of Thermal Gradient on Free Vibration of Clamped Visco-Elastic Rectangular Plate with Linearly Thickness Variation in Both Directions," Meccanica, Vol. 43, No. 4, 2008, pp. 449- 458.

[11] A. K. Gupta, A. Kumar and Y. K. Gupta, "Vibration of Visco-Elastic Parallelogram Plate with Parabolic Thickness Variation," Applied Mathematics, Vol. 1, No. 2, 2010, pp. 128-136.

[12] A. K. Gupta and P. Singhal, "Effect of Non-Homogeneity on Thermally Induced Vibration of Orthotropic ViscoElastic Rectangular Plate of Linearly Varying Thickness," Applied Mathematics, Vol. 1, No. 4, 2010, pp. 326-333. 